\title{
Lung liquid clearance in preterm lambs assessed by magnetic resonance imaging
}

\author{
Ali Houeijeh ${ }^{1,2}$, Pierre Tourneux ${ }^{1,3}$, Sébastien Mur ${ }^{4}$, Estelle Aubry ${ }^{1,5}$, Romain Viard ${ }^{6}$, Dyuti Sharma ${ }^{1,5}$ and Laurent Storme ${ }^{1,4}$
}

BACKGROUND: Postnatal adaptation requires liquid clearance and lung aeration. However, their relative contribution to the expansion of functional residual capacity (FRC) has not been fully investigated. We studied evolution of lung liquid removal and lung aeration after birth in preterm lambs.

METHODS: Lung liquid content and lung volume were assessed at birth and every 30 min over $2 \mathrm{~h}$ using magnetic resonance imaging (MRI) in three groups of lambs delivered by cesarean: preterm, late preterm, and late preterm with antenatal steroids. Lung function and mechanics of the respiratory system were also measured.

RESULTS: Lung liquid content increased by approximately 30\% in the preterm group $(P<0.05)$, whereas it did not change significantly in the late preterm lambs. Antenatal steroids induced a 50\% drop in the lung liquid content $(P<0.05)$. Total lung volume increased in all groups $(P<0.05)$ but was higher in the late preterm + steroids group relative to other groups $(P<0.05)$. Compliance and resistances of the respiratory system were significantly correlated with lung liquid content $(P<0.05)$. CONCLUSION: FRC expansion results mainly from an increase in lung volume rather than a decrease in lung liquid in preterm and late preterm lambs. Antenatal steroids promote FRC expansion through increases in lung volume and liquid clearance.

F etal airways are filled with liquid produced by the epithelial cells of the distal airways. The transition to extrauterine life at birth requires rapid aeration of the lungs for initiating gas exchange (1). The airways and airspaces are rapidly cleared of liquid to allow the onset of air-breathing. Many studies have focused on the role of epithelial sodium channels in lung liquid reabsorption (2). Coupled with Na,K-ATPase (Na pump), $\mathrm{Na}$ channels facilitate trans-epithelial $\mathrm{Na}$ flux, thereby drawing water from the lung lumen into the interstitium, a process that normally begins during labor, and continues after birth. Ventilation and oxygenation promote lung liquid clearance through the epithelial cells toward the interstitium $(3,4)$. Recent studies have asserted that the transpulmonary pressure generated by inspiratory efforts also plays a critical role in airway liquid clearance $(5,6)$. During the first hours after the birth, lung liquid within the interstitium is transferred to the circulation via lymphatic and blood vessels (7).

Delayed lung liquid clearance is associated with impaired lung function. Preterm birth and elective cesarean section are associated with an increased risk for neonatal respiratory failure, resulting at least in part from the impaired clearance of lung liquid. Antenatal steroids are known to reduce the risk by promoting lung maturation and surfactant release (8).

Although lung aeration and liquid clearance are sequential but overlapping processes, their relative contributions to normal functional residual capacity (FRC) have not been fully investigated. The aim of this study was to assess the kinetics of lung liquid clearance and lung expansion in experimental models of preterm and late-preterm lambs. The effects of antenatal steroids were also investigated. Magnetic resonance imaging (MRI) was used to estimate serial changes in lung liquid content and lung volume.

\section{METHODS}

\section{Experimental Model}

All animal procedures and protocols used in this study were approved by the French "Ministère de l'Agriculture, de la Pêche et de l'Alimentation" before the studies were conducted and were carried out in the Department of Experimental Research of our Hospital centre (animal facility agreement $n^{\circ} 59286$ ). Pregnant ewes of the Colombia-Rambouillet breed were housed in individual pens starting a week before (at $110 \mathrm{~d}$ of gestational age) and throughout the procedure.

Sixteen pregnant ewes were randomly assigned to three groups: preterm $(n=5)$, late preterm $(n=5)$, and late preterm with antenatal steroids $(n=6)$. Forty-eight hours before the surgery, the ewes received normal saline or $0.5 \mathrm{mg} / \mathrm{kg}$ of Dexamethasone (Qualimed, Puteaux, France) twice daily via an intramuscular injection. The fetal sheep underwent in utero surgery at a gestational age of 122-124 d (preterm, $n=5$ ), or 134-136 d (late preterm, $n=11$ ) after fasting for $24 \mathrm{~h}$ (full term: $146 \mathrm{~d}$ ). The ewes were sedated with intravenous sodium pentobarbital (total dose, $2-4 \mathrm{~g}$ ) and anesthetized with $0.25 \%$ bupivacaine hydrochloride $(4 \mathrm{ml})$ by a lumbar injection. The ewes were kept sedated but breathed spontaneously throughout the surgery and the experiment. Under sterile conditions, the fetal lambs' necks were delivered through a uterine incision. The fetus heads were placed immediately into a tight sterile pouch to prevent pulmonary ventilation. A median skin incision was made after a local infiltration

\footnotetext{
"EA4489 "Perinatal Environment and Health", FHU “1000 days 4 Health", University of Lille - Nord de France, Lille, France; ${ }^{2}$ Pediatric Cardiology Unit, Department of Pediatrics, Lille University Hospital, Lille, France; ${ }^{3}$ Department of Neonatology, Maternity, Amiens University Hospital, Amiens, France; ${ }^{4}$ Department of Neonatology and Maternity, Jeanne de Flandre Hospital, Lille University Hospital, Lille, France; ${ }^{5}$ Pediatric Surgery Unit, Department of Pediatrics, Lille University Hospital, Lille, France; ${ }^{6}$ Plateforme Imagerie du Vivant, University of Lille, Nord de France, Lille, France. Correspondence: Laurent Storme (laurent.storme@chru-lille.fr)

Received 5 April 2016; accepted 30 November 2016; advance online publication 3 May 2017. doi:10.1038/pr.2017.31
} 
with lidocaine ( $2 \mathrm{ml}, 1 \%$ solution) and analgesia by Nubain (Mylan, Canonsburg, Pennsylvania) $10 \mathrm{mg}$ by subcutaneous injection. A polyvinyl catheter $(20 \mathrm{G})$ was advanced into the superior vena cava through the jugular vein. Anesthesia of the fetal lamb was then performed through this catheter with sufentanyl (infusion rate $=2 \mu \mathrm{g}$ / $\mathrm{min}$ ) and pancuronium (infusion rate $=400 \mu \mathrm{g} / \mathrm{h}$ ). A polyurethane orotracheal cuffed tube $(3.5 \mathrm{~mm}$ diameter $)$ was introduced by oral intubation. Air leaks were carefully controlled and prevented by adapting the pressure of the cuff. The tracheal tube was occluded until the mechanical ventilation began. Special care was taken to maintain the body temperature of the lambs between 38 and $39^{\circ} \mathrm{C}$ along the study period. The lambs were dried carefully before placing them in the head coil of the MRI. Warm and dry blankets were placed inside the head coil and covered the lambs. The blankets were changed regularly during the experiments

\section{Protocol}

The ewe was placed on the examination table of the MRI system. The fetal lamb was extracted and placed in the head coil (quadrature coil, $250 \mathrm{~mm}$ diameter, $270 \mathrm{~mm}$ length) in a head-first prone position. The umbilical cord was not clamped to maintain feto-placental circulation during the period of installation in the head coil. The ewe lay in a supine position near the head coil to prevent any stretching of the umbilical cord. MR images were obtained with an open magnet 0.2 Tesla MR scanner (Hitachi Medical Systems, Airis, Tokyo, Japan; maximum gradient strength $15 \mathrm{mT} / \mathrm{m}$, slew rate $30 \mathrm{mT} / \mathrm{m} / \mathrm{ms}$ gradients). The tracheal tube was connected to a servo 900C respirator suitable for MRI (Siemens-Elema, Solna, Sweden). Ventilation was started for $120 \mathrm{~min}$. The umbilical cord was clamped at $30 \mathrm{~min}$ after starting mechanical ventilation. MRI acquisition was then performed every $30 \mathrm{~min}$.

Fast Spin Echo $\mathrm{T}_{2}$-weighted sequences (repetition time/echo time $=$ $2,938 / 100 \mathrm{~ms}$, flip angle $=90$ degree, echo train length $=18$, NEX $=1$, acquisition duration $=35 \mathrm{~s}$, bandwidth $=34 \mathrm{kHz}$, field of view $=320 \times 320 \mathrm{~mm}^{2}$, acquisition matrix $=128 \times 256,12$ contiguous slices with $10 \mathrm{~mm}$ thickness) were used. This Fast Spin Echo sequence has two main characteristics: (i) it provides a signal proportional to the proton density. Hence, it facilitates a high visualization of both the amount of pulmonary liquid (high signal intensity) and of the lack of liquid when the lung fills up with air (low signal intensity) in a short time; and (ii) it is a relative black blood sequence, thus avoiding blood flow-related signal $(9,10)$.

Concerning the MRI acquisition, images were obtained along three orthogonal directions (transaxial, coronal, and sagittal) at the beginning of experiments. Unfortunately, three orthogonal acquisition time is $3.5 \mathrm{~min}$ which is physiologically incompatible with an apnea every $30 \mathrm{~min}$. Therefore, interval measurements were obtained in the sole coronal direction during $35 \mathrm{~s}$ apnea. Then, we calculated a correcting factor taken into account the ratio of pulmonary volume assessed by three orthogonal directions/pulmonary volume assessed by coronal direction only. Pulmonary volumes obtained by coronal direction were adjusted using this correcting factor which increase the accuracy of the lung volume measurement as previously described (11) (Figure 1). While unclamping the tracheal tube $\left(\mathrm{T}_{0}\right)$, initial respirator settings were set in a pressure controlled mode as follows: peak inspiratory pressure: $22 \mathrm{~cm} \mathrm{H}_{2} 0$; positive end expiratory pressure: $4 \mathrm{~cm} \mathrm{H} \mathrm{H}_{2} 0$; inspiratory time: $0.5 \mathrm{~s}$; and ventilatory rate: $40 / \mathrm{min}$. The ventilatory rate and peak inspiratory pressure were adjusted as necessary according to the protocol in (Table 1). $\mathrm{FiO}_{2}$ was set to maintain SpO2 between 92 and $98 \%$. At $30 \mathrm{~min}$ from the onset of mechanical ventilation (T30), and then every $30 \mathrm{~min}$ until T120, successive acquisitions were obtained during an expiratory apnea of $35 \mathrm{~s}$ by pushing the expiratory pause button on the ventilator. Thus, positive end expiratory pressure was maintained along the period of apnea in order to prevent lung derecrutment during expiratory pause. The MR sequences were configured in a dynamic mode. In that manner, the radio frequency gains, as well the emitter for the receiver amplifiers, remained unchanged during the whole $2 \mathrm{~h}$ experiment, and the image grey levels were comparable from one acquisition to another. The umbilical cord was clamped at $\mathrm{T}_{30}$. At the end of the experiment $\left(\mathrm{t}_{120}\right)$, images were acquired along three orthogonal directions (Figure 1).
Additional physiological parameters were recorded. Tidal volume $\left(V_{\mathrm{t}}\right)$ and ventilatory pressures were continuously monitored with a flow and pressure transducer placed between the tracheal tube and the breathing circuit (pneumotachograph monitor 2100 Eurocare, West Yorkshire, UK) and stored in a computer (PhysioTrace software, CIC-IT 807, Lille, France). Static compliance of the respiratory system expressed per kilogram was calculated as the tidal volume divided by the difference between inspiratory plateau pressure obtained by pushing the inspiratory pause button on the ventilator for $5 \mathrm{~s}$ and the expiratory pressure obtained by pushing the expiratory plateau pressure for $5 \mathrm{~s}(12,13)$. Blood samples were drawn for blood gas analysis every 60 min (iSTAT system, ABBOTT, Chicago, IL).

Change in lung liquid content was assessed from MRI analyses as previously described (11). Briefly, image processing was performed by using specific imaging software (ArtiMED, University Lille2, Lille, France). Total pulmonary volumes representing gas + liquid volumes, were free-form contoured by a blinded investigator, while analyzing slices sequentially according to the successive acquisition times. The different regions of interest obtained at a given time on individual consecutive sections have been merged by a fuzzy logic data fusion algorithm to minimize the partial volume effects. Slices had a distance of $10 \mathrm{~mm}$ to avoid signal artifacts from the blood perfusion. From these contours and volumes, the following parameters were studied as a function of time: lung volume, lung liquid content and functional residual capacity, and lung liquid clearance. Pulmonary liquid content change was assessed by computing the change in the gray intensity of the signal in the contoured zones. Assuming that the content of the lungs is either liquid or air and that the lung is filled of liquid with no air at T0, pulmonary liquid density was considered as the ratio of the pulmonary liquid to lung volume. This method was previously found to be accurate at assessing change in lung volume and lung liquid content after birth (11).

\section{Statistics}

The statistical analysis was performed by an independent statistician using SPSS 2012 software (SPSS, Chicago, IL). Quantitative measurements were expressed as the mean \pm standard deviation. Data were analyzed using the Friedman test (Applied Nonparametric Statistics, 2nd ed, Boston) when significant, subgroups were analyzed with the Wilcoxon test. Linear relationships were studied by the Spearman bidirectional test. Qualitative data are expressed as percentages. A $P$ value $<0.05$ was considered significant.

\section{RESULTS}

Birth weights were 2,450 $\pm 230 \mathrm{~g}, 3,450 \pm 800 \mathrm{~g}$, and 3,200 $\pm 650 \mathrm{~g}$ in the preterm, late preterm, and late preterm + steroids groups $(P<0.05)$, respectively.

\section{Total Lung Volume and Lung Liquid}

Total lung volumes measured by MRI were considered the lung liquid volume content at that time point (T0) and were $38 \pm 4$, $42 \pm 4$, and $45 \pm 5 \mathrm{ml} / \mathrm{kg}$ in the preterm, late preterm, and late preterm + steroids groups, respectively $(P=0.15)$. Total lung volume measured by MRI increased similarly from T0 to T120 by $45 \%$ in each group $(P<0.05)$ (Figure 2$)$. However, the lung volume expressed in kilogram was higher in the late preterm + steroids group than in the preterm group at T30, T60, T90, and T120 $(P<0.05)$ (Figure 2). The lung liquid content did not change during the study period in the late preterm group, decreased by $55 \%$ in the late preterm + steroids group, and increased by $28 \%$ in the preterm group $(P<0.05)$ (Figure 2 ). FRC rose sharply in the late preterm + steroids group, reaching a level more than fivefold that of the FRC in the preterm group at the 30th minute and at all subsequent time points $(P<0.001)$. Similarly, FRC creation was less important in the 


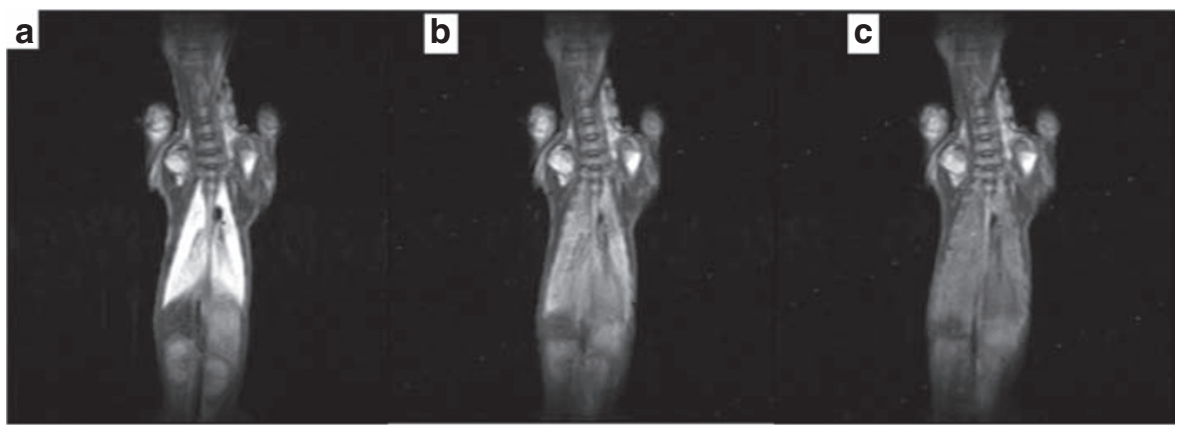

Figure 1. An example of sequential magnetic resonance imaging on a coronal slice. Images (a) before the first breath; (b) $1 \mathrm{~h}$; and (c) $2 \mathrm{~h}$ after starting ventilation. Liquid content within the lungs appears with a high signal before the first breath and decreases during ventilation throughout the study period.

Table 1. Protocol for modifying the ventilator settings according to $\mathrm{PaCO}_{2}$ values

\begin{tabular}{ll}
\hline $\mathrm{PaCO}_{2}(\mathrm{mmHg})$ & Ventilator settings \\
\hline$<25$ & Decrease RR by 10 and PIP by $5 \mathrm{cmH}_{2} \mathrm{O}$ \\
$25-35$ & $\begin{array}{l}\text { Decrease PIP by } 5 \mathrm{cmH}_{2} \mathrm{O} \text { or decrease RR by } 10 \\
\text { if PIP }=20 \mathrm{cmH}_{2} \mathrm{O}\end{array}$ \\
$35-50$ & No modification \\
$50-70$ & Increase RR by 20 or increase PIP by $5 \mathrm{cmH}_{2} \mathrm{O}$ if RR $=80$ \\
$>70$ & Increase RR by 20 and PIP by $5 \mathrm{cmH}_{2} \mathrm{O}$ \\
\hline
\end{tabular}

$\mathrm{PIP}$, peak inspiratory pressure; $\mathrm{RR}$, respiratory rate, breath/min.

late preterm compared to the late preterm + steroids group after 30 min of ventilation $P<0.05$ (Figure 3).

\section{Lung Function}

After positioning the fetal lambs in the MRI head coil (intact cord), and just before starting mechanical ventilation, blood gases, lactate concentrations, $\mathrm{SpO}_{2}$ and heart rate were similar in the three groups (Table 2). $\mathrm{FiO}_{2}$, respiratory rate and peak inspiratory pressure were higher in the preterm group than in the two other groups $(P<0.05)$ (Table 2). $\mathrm{PaO} 2 / \mathrm{FiO} 2$ was higher in the group late-preterm + steroids than in the two other groups (Table 2). Despite the increase in ventilator settings, the arterial $\mathrm{pH}$ and $\mathrm{PaO} 2$ were lower, and the $\mathrm{PaCO} 2$ and lactate concentrations were higher in the preterm group than in the two other groups after starting mechanical ventilation $(P<0.05)$ (Table 2).

\section{Respiratory Mechanics}

Tidal volume increased during the study period in the late preterm group $(P<0.05)$, but did not change in the preterm and late preterm $+\mathrm{GC}$ groups (Figure 4). Static compliance of the respiratory system was higher in the late preterm + GC group than in the two other groups at all-time points $(P<0.05)$ (Figure 5). Static compliance of the respiratory system was lower in the preterm group than in the two other groups at all time points $(P<0.05)$ (Figure 5). Resistances of the respiratory system decreased steadily in the late preterm groups $(P<$ $0.05)$ (Figure 5). The resistances were lower in the late preterm groups than in the preterm group $(P<0.05)$ (Figure 5). Static
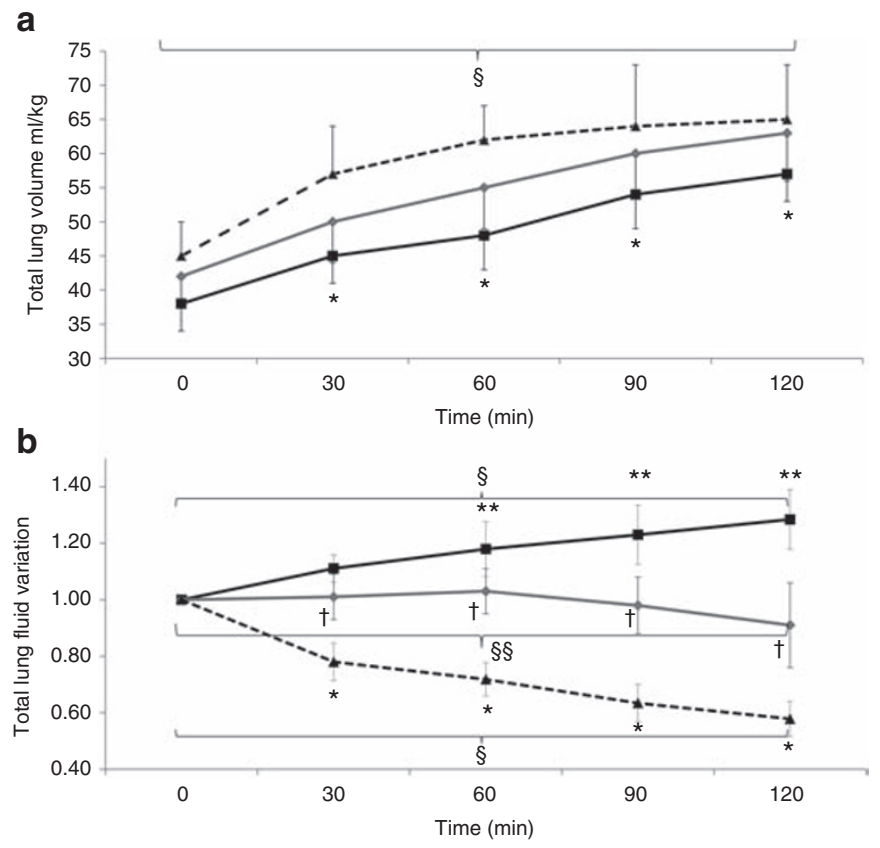

Figure 2. (a) Total lung volume increased in the three groups $(P<0.05)$. The upward trend was markedly higher in late preterm + steroids group (dotted line) than in preterm group (black solid line) and late preterm group (gray line). (b) Total lung fluid content did not change significantly in late preterm group (gray line) $(\S \S P=0.17)$. Total lung fluid content increased gradually by approximately $30 \%$ in preterm group (black solid line) and decreased in the late preterm + steroids group (dotted line). Results are expressed as mean \pm SD. ${ }^{*} P<0.05$ between preterm and late preterm + steroids groups. ${ }^{* *} P<0.05$ between late preterm and preterm groups. ${ }^{\dagger} P<0.05$ between late preterm and late preterm + steroids groups. ${ }^{\S} P<0.05$ changes in total lung fluid or total lung volume between the beginning of experiments and the end at $120 \mathrm{~min}$.

compliance of the respiratory system was correlated with the gas volume of the lung and inversely correlated with the lung liquid content $(P<0.05)$ (Figure 6). Resistances of the respiratory system were correlated with lung liquid content and inversely correlated with the gas volume of the lung $(P<0.05)$ (Figure 6).

\section{DISCUSSION}

Postnatal adaptation at birth requires the simultaneous liquid clearance and aeration of the lungs (6). Mechanisms of 
liquid shifts between lung lumen, interstitial space, lymphatic and blood vessels have been previously studied (4). Lung liquid clearance is triggered during labor by the surge in fetal catecholamines and glucocorticoids which increase sodium channels expression and Na-K-ATPase activity. After birth, respiratory movements become the driver force of liquid clearance $(1,6)$. Thus alveolar liquid is cleared quickly after birth

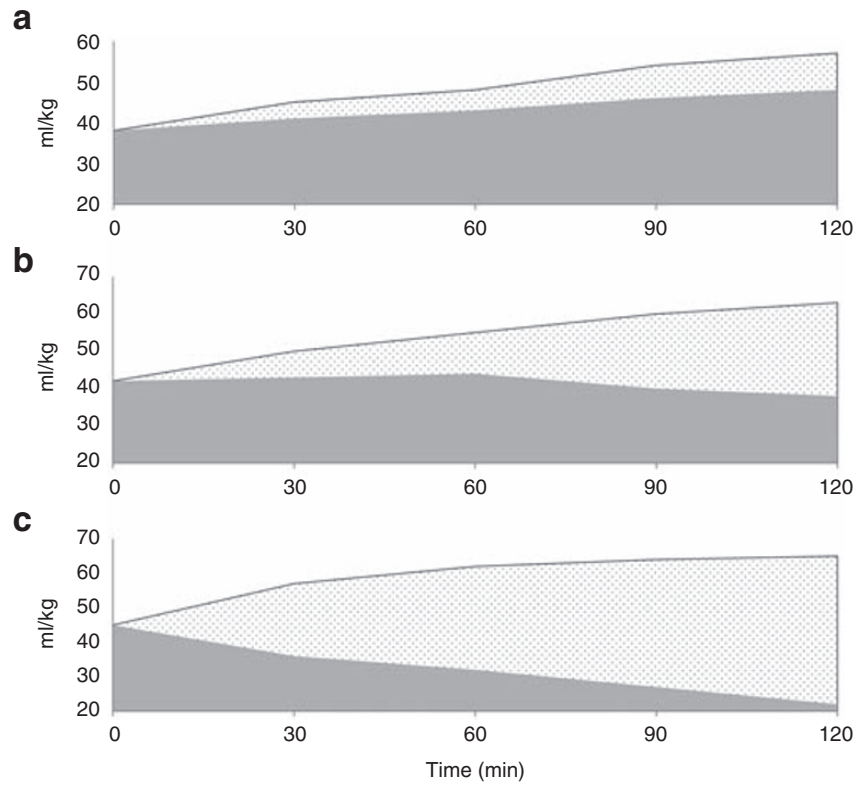

Figure 3. (a) Estimation of functional residual capacity (FRC) in preterm group. (b) Estimation of FRC in late preterm group. (c) Estimation of FRC in late preterm + steroids lambs. Dotted area represents FRC. Shaded area represents total lung liquid measured by MRI. FRC expansion is impaired in preterm lambs and to a lesser extent in late preterm lambs compared to late preterm group + steroids lambs. Volumes are expressed as $\mathrm{ml} / \mathrm{kg}$. to the interstitium, before its resorption over the next hours through the lymphatic system and the pulmonary vessels. The increase in the pulmonary interstitial pressure after birth supports this phenomenon (14). During this time of transition at birth, the lungs and the chest wall have to expand to accommodate the increase in FRC and the interstitial liquid (6). The relative contribution of liquid clearance and increase in lung volume to the development of the FRC has not been fully studied. Immaturity and surfactant deficiency in the premature infant is associated with delayed lung liquid clearance and poor aeration of the lungs (15). We compared the changes in total lung volume and lung liquid content in very preterm and late-preterm lambs treated or not treated with antenatal steroids. We found that the FRC expansion mainly results from an increase in total lung volume rather than a decrease in lung liquid content in late preterm lambs within the first hours after birth. In the immature preterm lambs, lung liquid content increased markedly during early life. Antenatal steroids promote both early liquid clearance from the lungs and FRC elevation. Respiratory mechanics are highly correlated with lung liquid content.

The main findings in our study are the simultaneous and serial measurements of both lung liquid content and FRC using MRI and lung function measurements. Our results highlight the major differences in the progression of liquid clearance and FRC development according to gestational age and treatment with antenatal steroids. The newborn lambs have been investigated at two different lung maturational ages, corresponding to $26-28$ and $34-36$ wk of gestation in human beings (16). Surprisingly, in the more immature lambs, total lung liquid content rose rapidly after birth. As FRC creation results from postnatal lung expansion and liquid clearance, liquid accumulation impedes FRC expansion. This phenomenon

Table 2. Serial blood gases in preterm $(n=5)$, late preterm $(n=5)$, and late preterm + steroids $(n=6)$ newborn lambs before (T0) and 60 and 120 min after starting mechanical ventilation (T60 and T120)

\begin{tabular}{|c|c|c|c|c|c|c|c|c|c|}
\hline & \multicolumn{3}{|c|}{ TO } & \multicolumn{3}{|c|}{ T60 } & \multicolumn{3}{|c|}{ T120 } \\
\hline & Preterm & Late preterm & $\begin{array}{l}\text { Late preterm } \\
\text { + steroids }\end{array}$ & Preterm & Late preterm & $\begin{array}{l}\text { Late preterm } \\
\text { + steroids }\end{array}$ & Preterm & Late preterm & $\begin{array}{l}\text { Late preterm } \\
+ \text { steroids }\end{array}$ \\
\hline $\mathrm{SpO}_{2}$ & $48 \pm 12$ & $55 \pm 9$ & $53 \pm 12$ & $85 \pm 17^{*}$ & $94 \pm 5$ & $95 \pm 3$ & $78 \pm 19^{*}$ & $94 \pm 3$ & $94 \pm 2$ \\
\hline $\mathrm{FiO}_{2}$ & - & - & - & $100 \pm 0^{*}$ & $36 \pm 8$ & $25 \pm 4$ & $100 \pm 0^{*}$ & $26 \pm 3$ & $21 \pm 0$ \\
\hline Vt & - & - & - & $4 \pm 1^{*}$ & $7 \pm 2$ & $11 \pm 4$ & $6 \pm 2^{*}$ & $11 \pm 3$ & $12 \pm 4$ \\
\hline PH & $7.32 \pm 0.1$ & $7.35 \pm 0.02$ & $7.36 \pm 0.03$ & $7.11 \pm 0.1^{*}$ & $7.35 \pm 0.06$ & $7.43 \pm 0.08$ & $7.15 \pm 0.2^{*}$ & $7.32 \pm 0.06$ & $7.39 \pm 0.06$ \\
\hline $\mathrm{PaO}_{2}$ & $20 \pm 7$ & $23 \pm 4$ & $21 \pm 3$ & $35 \pm 20$ & $46 \pm 6$ & $44 \pm 5$ & $29 \pm 17$ & $42 \pm 8$ & $44 \pm 5$ \\
\hline $\mathrm{PaCO}_{2}$ & $51 \pm 7$ & $52 \pm 6$ & $51 \pm 6$ & $89 \pm 25^{*}$ & $45 \pm 6$ & $39 \pm 10$ & $75 \pm 33^{*}$ & $47 \pm 9$ & $47 \pm 12$ \\
\hline
\end{tabular}

$\mathrm{HR}$, heart rate in beats/min; $\mathrm{SpO}_{2}$, transcutaneous $\mathrm{O}_{2}$ saturation in \%; $\mathrm{FiO}_{2}$, fraction of inspiratory $\mathrm{O}_{2}$ in \%; RR, Respiratory Rate in breath/min; $\mathrm{PIP}$, Peak Inspiratory Pressure in cmH2O; $\mathrm{Vt}$, tidal volume in $\mathrm{ml} / \mathrm{kg} ; \mathrm{PaO}_{2}$ and $\mathrm{PaCO}_{2}$ expressed in $\mathrm{mmHg}$; Bicarb, bicarbonate concentration in $\mathrm{mmol} /$; lactate, blood lactate concentration in mmol/I. Values are the means $\pm \mathrm{SD}$. ${ }^{*} P<0.05$ compared to the other groups at each time point. 


\section{Articles | Houeijeh et al.}

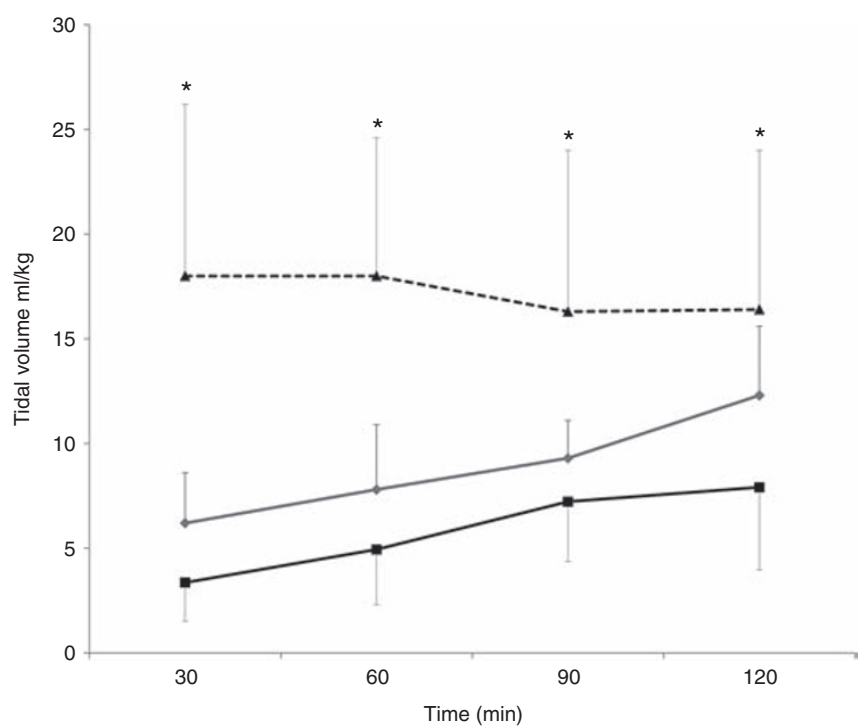

Figure 4. Tidal volume $(\mathrm{Vt}, \mathrm{ml} / \mathrm{kg})$ changes after starting ventilation in Preterm group (black solid line), late preterm group (gray line), and late preterm + steroids group (dotted line). Vt was higher in late preterm lambs + steroids than in preterm and late preterm lambs. Results are expressed as mean \pm SD. ${ }^{*} P<0.05$ between the preterm and late preterm lambs + steroids groups.

explains at least in part the impaired lung function and respiratory mechanics. Pulmonary liquid rise could be explained mainly by the pulmonary edema secondary to respiratory failure. Severe hypoxia may increase lung vascular filtration pressure and thereby promote trans-vascular fluid filtration. Furthermore, impaired lung function and respiratory mechanics required high ventilator pressures and $\mathrm{FiO}_{2}$. The resulting parenchymal barotrauma and oxidative stress-induced lung injury may have in turn enhanced the vascular leak and promoted lung liquid accumulation. Previous study reported that the extravascular lung liquid content measured by the labeled indicator dilution method was twofold higher in preterm than late preterm lambs mechanically ventilated for $3 \mathrm{~h}$ (15). Similarly, the pulmonary lymph flow is twice as high in preterm compared to term lambs, suggesting an increase in lung vascular permeability (17). Our findings are in accordance with these studies, and provide additional information on the kinetics of the events. Several other mechanisms may contribute to the early rise in lung liquid content in preterm lambs. Mainly the decrease of the expression of sodium channels, Na,KATPase activity, as well as surfactant synthesis in immature lung (18-20). Moreover, these mechanisms are more deficient in case of cesarean prior labor $(4,21)$. In addition, the severe hypoxic respiratory failure may elevate pulmonary vascular resistances, increasing filtration pressure, interstitial fluid content and lymph flow (22). However, previous studies reported a reduction of the extravascular lung liquid content in some experimental preterm models $(15,20)$. It is noteworthy that the degree of prematurity as evidenced by the severity of the hypoxic respiratory failure was far much greater in our experimental model of preterm lambs (122-124 d gestation) than the previous studies. Otherwise, this apparent discrepancy might a
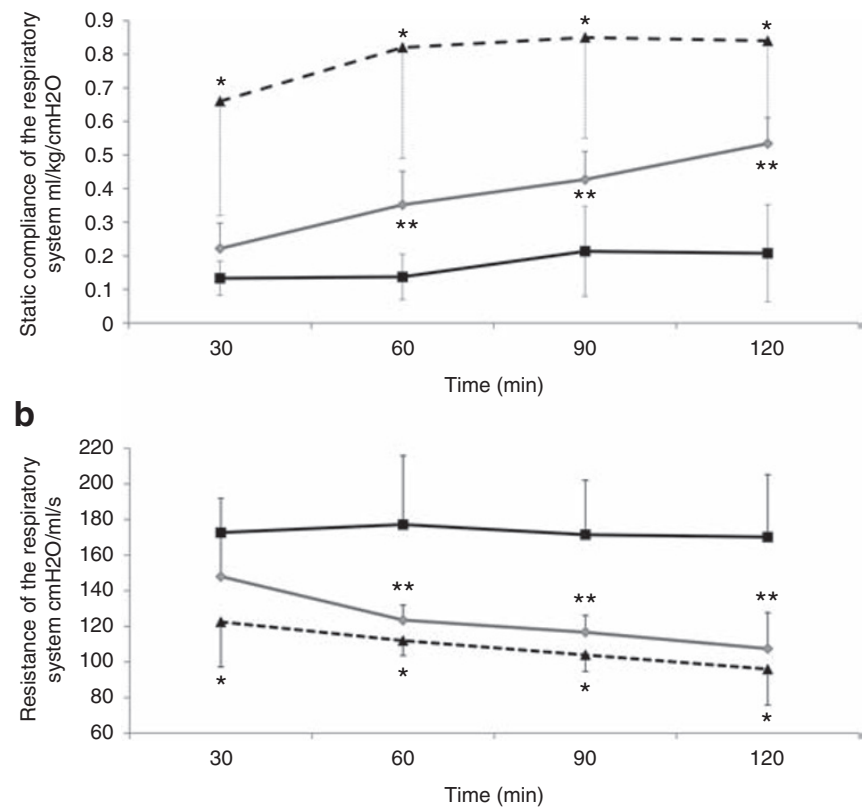

Figure 5. (a) Changes in the static compliance of the respiratory system. Static compliance was significantly higher in late preterm+ steroids group (dotted line) than in preterm (black solid line) and late preterm (gray line) groups at all times. (b) Changes in the resistance of the pulmonary system. Resistance was higher in preterm group (black solid line) at any time point than in late preterm (gray line), and late preterm + steroids (dotted line) lambs. Results are expressed as mean \pm SD. ${ }^{*} P<0.05$ between preterm and late preterm + steroids groups. ${ }^{* *} P<0.05$ between late preterm and preterm groups.

reflect species differences (rabbits vs. lambs), differences in experimental design (spontaneous breathing vs. mechanical ventilation while anesthetized and paralyzed), or methodological differences (gravimetric assay vs. MRI). Respiratory failure, atelectasis, lung edema, and pulmonary vasoconstriction may increase the MRI signal in area with low blood flow velocities that may contribute to a slight overestimation of lung fluid content. Besides, MRI cannot differentiate intraluminal liquid from interstitial and pleural fluid.

In late preterm lambs, the amount of lung liquid did not change during the first $2 \mathrm{~h}$ after birth, although the total lung volume increased steadily during this period. These findings indicate that FRC results mainly from progressive lung expansion rather than liquid clearance. Previous studies using phase-contrast $\mathrm{x}$-ray imaging in newborn rabbits determined that respiratory movements change transpulmonary pressure, which moves the alveolar lung liquid to the interstitial tissue (4-6). Antenatal steroids significantly modified the evolution of lung liquid clearance in the late preterm lambs. The total amount of lung liquid decreased as early as $30 \mathrm{~min}$ after starting ventilation and dropped by $50 \%$ at $120 \mathrm{~min}$. Thus, antenatal steroids accelerate FRC expansion through both an increase in lung volume and liquid removal. These results suggest that the lungs are still immature in the late preterm lambs with a relative deficiency of surfactant and sodium channels protein expression. Recent clinical trial in human being highlighted 


\section{Fluid clearance in the immature lung. $\quad$ Articles}

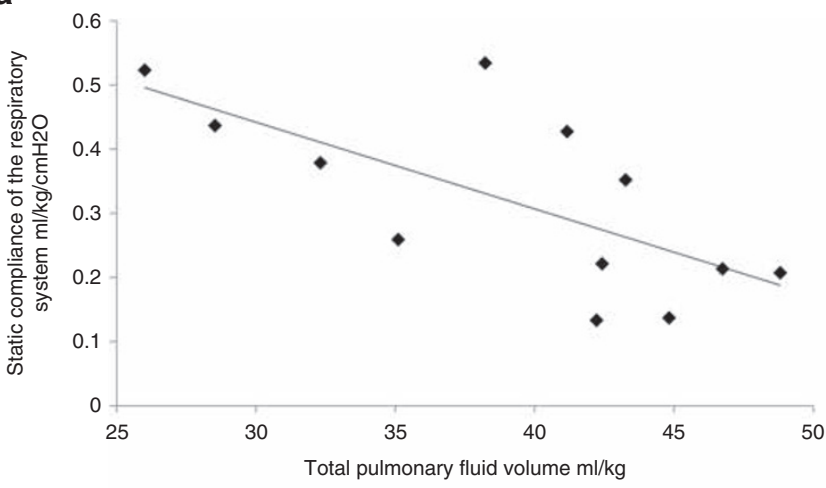

b

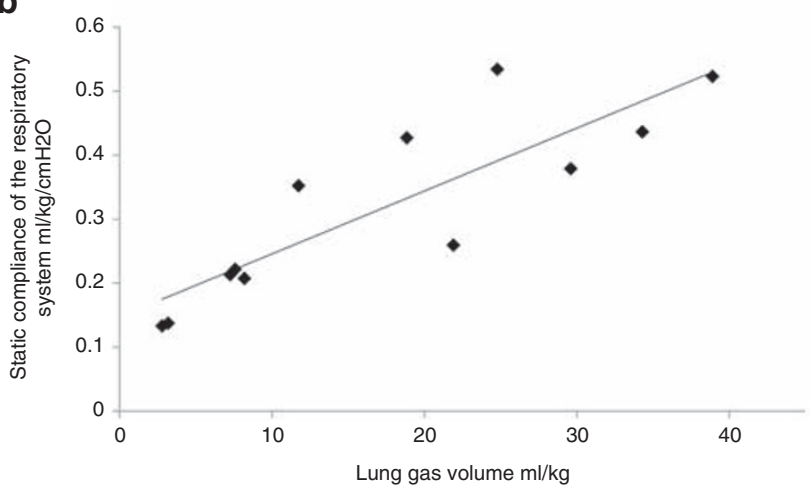

C

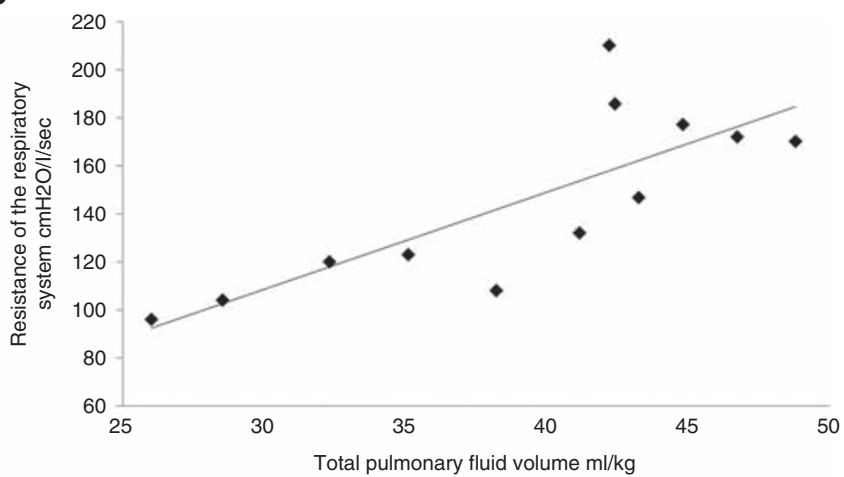

d

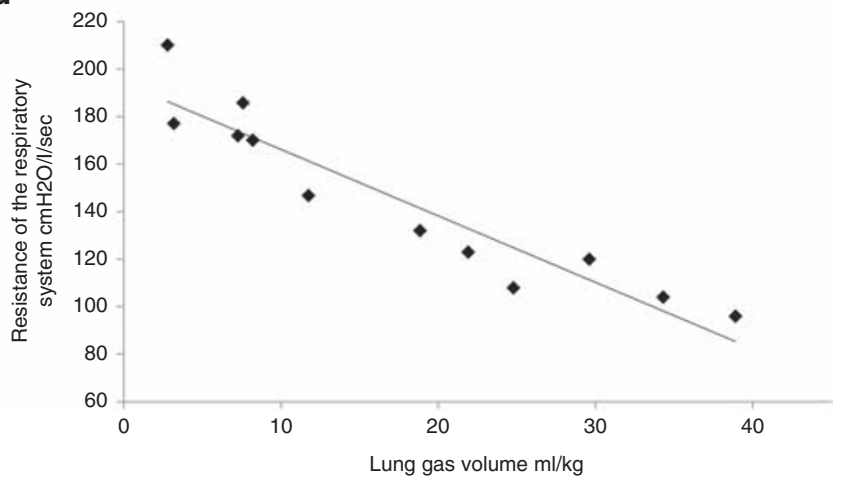

Figure 6. Correlation between total lung volume and total lung fluid content and both compliance and resistance of the respiratory system. (a) Static compliance was inversely correlated to the total lung fluid $(P=0.024, r=0.226)$. (b) Static compliance was directly correlated to lung gas volume $(P=0.023, r=0.288)$. Inversely, (c) the resistance of the respiratory system was directly correlated to the total lung fluid $(P<0.001, r=0.47),(\mathbf{d})$ whereas the relation with the lung gas volume was negative $(P<0.001, r=0.61)$.

the higher risk of respiratory morbidities in late preterm newborn not treated prenatally by steroids comparing to the treated group (23). Elective cesarean section before labor may have further delayed lung liquid clearance and impaired respiratory mechanics. The incidence of respiratory failure is increased by 10 -fold in cases of elective caesarian section before $38 \mathrm{wk}$ compared to vaginal delivery after $39 \mathrm{wk}$ (24), supporting the role of labor in lung liquid clearance and lung function at birth. Antenatal steroids reduce the risk of respiratory failure in this condition (25). Our results provide additional information about this mechanism by showing that elective cesarean section before labor alters the kinetics of lung liquid clearance, respiratory mechanics and FRC creation, which can be prevented at least in part by antenatal steroids.

Our results show that both lung liquid content and FRC play a major role in respiratory functions and mechanics. In particular, compliance of the respiratory system increases with FRC. A similar association has been reported previously in preterm lambs (26). We further show that compliance of the respiratory system is also dependent on the total amount of liquid remaining within the lung. A similar relationship was found between lung liquid content and FRC and resistances of the respiratory system. Resistances are related to the diameter of the airways (airway resistance) and to the tissue resistance, which account for about $40 \%$ of total resistance in newborns owing to higher parenchymal density. Tissue resistances depend on the friction forces that occur between the gas stream and the respiratory system and on the viscosity of the lung parenchyma (27). As the viscosity of the liquid is considerably higher than air, tissue resistance is related to lung liquid content (5). Our findings provide additional evidence that lung liquid clearance play a role in the change in resistance of the respiratory system after birth. Although the lung liquid content influences gas exchange, oxygenation, and carbon dioxide exchange can be sustained even when the lung liquid content is still elevated, as we observed in late preterm group, and in previous reports (26). It is likely that effective gas exchange starts as soon as part of the airspaces is cleared, even though the liquid is stored within the lung interstitium.

Several methods were used to study lung liquid clearance. MRI has the advantage to be able to perform simultaneous and serial assessment of both of liquid content and gas volume. In vitro studies found MRI accurate to assess lung liquid content. An excellent agreement between MRI assessment of lung liquid and gravimetric wet/dry ratio has been found in piglet lungs, with correlation factor of 0.99 , and an average mean error of $1.8 \%$ (28-31). In vivo studies confirmed the accuracy of MRI measurement of lung fluid (32). A correlation coefficient of 0.91 between the MRI and gravimetric measurements was found in a rat model, the average mean error being around 10\% (32). This good correlation was confirmed in numerous other experimental studies. In a model of oleic acid-induced lung injury in dogs, 
MRI was found sensitive enough to measure slight changes in lung liquid and to assess the time course of the edema formation (33). In rats with experimental ventilator-induced lung injury, the degree of liquid accumulation could be quantified by MRI (34). Furthermore, in human being, MRI has been used also to estimate lung water content in preterm infants with or without bronchopulmonary dysplasia $(35,36)$ or in patients with cystic fibrosis (37). It is noteworthy to outline that most other methods showed equivalent or less significant correlation. For instance, indicator dilution method overestimates lung fluid content by $10-15 \%$, whereas Positron Emission Tomography underestimates lung fluid content by $10-15 \%$ (38).

Our study has some limitations. MRI requires cesarean section and paralysis of the lambs to ensure careful positioning in the head coil before the first breath. The umbilical cord was kept intact during this procedure and the first $30 \mathrm{~min}$ of ventilation to prevent hypoxia. Moreover, prolonged apnea is needed during the imaging acquisition. These technical limitations may impact liquid clearance as previously reported $(18,39,40)$. They preclude exploration of lung liquid clearance at birth in more physiological conditions such as vaginal delivery and spontaneous breathing. Otherwise, MRI technique might slightly overestimate lung liquid content because of a possible residual signal of the dry lungs and the blood in small vessels. Although the MRI signal used in the present study is roughly proportional to the proton density and therefore water content, the lung parenchyma which contains few concentrations of fat and other hydrogen-bound complexes may not be completely "black" on MRI leading to a slight overestimation of the lung water content. Besides, its effectiveness in suppressing blood signal has been assessed in large vessels where blood flow velocity is elevated. We cannot exclude that this velocitysensitive sequence might fail to remove the residual slow-flow blood signal induced within the smaller vessels. However, in a previous study (11), as well as in the present study, the lung signal intensity can decrease by a factor 10 , indicating that the eventual residual blood flow signal contributes to less than $10 \%$ of the lung signal. Similar results were reported in normal human lung. In this study, the lung signal intensity was similar to the background noise when the echo time was greater than $2.5 \mathrm{~ms}$, which is the case in our MRI sequence (9). In another experimental study in dogs, the signal intensity was not correlated to pulmonary blood flow measured by microsphere technique (33). Additionally, MRI cannot differentiate delayed liquid clearance from lung injury-induced water accumulation through atelectasis or inflammation. Indeed, the size of the voxel is too large to determine the location of liquid within the lung parenchyma, i.e., intra- or extraluminal. In the same way, the signal of an area of atelectasis is related to its water content and not to the atelectasis itself. Finally, additional experiments should be done to understand the mechanisms by which MRI signal intensity increased with time in the preterm lambs.

\section{CONCLUSION AND PERSPECTIVES}

Transition at birth requires rapid FRC creation. FRC expansion results from the simultaneous expansion of the lung and lung liquid clearance. In the case of delayed liquid clearance, lung aeration depends mainly on an increase in lung volume. In the most immature lung, surfactant deficiency and lung injury are associated with liquid accumulation within the lung, limiting FRC creation. This phenomenon clearly contributes to poor lung function and respiratory mechanics. Alternatively, antenatal steroids can promote FRC creation at birth through a combination of accelerated lung liquid removal and lung expansion. The MRI technique still limited actually requiring non physiological conditions. Nevertheless, it seems very promising for the repeated evaluation of lung functions in the future.

\section{STATEMENT OF FINANCIAL SUPPORT}

This study received financial support from Lille University.

Disclosure: Authors have no conflicts of interest to declare.

\section{REFERENCES}

1. Olver RE, Walters DV, M Wilson S. Developmental regulation of lung liquid transport. Annu Rev Physiol 2004;66:77-101.

2. Siew ML, Wallace MJ, Allison BJ, et al. The role of lung inflation and sodium transport in airway liquid clearance during lung aeration in newborn rabbits. Pediatr Res 2013;73(4 Pt 1):443-9.

3. Bland RD. Loss of liquid from the lung lumen in labor: more than a simple "squeeze". Am J Physiol Lung Cell Mol Physiol 2001;280:L602-5.

4. Bland RD, Nielson DW. Developmental changes in lung epithelial ion transport and liquid movement. Annu Rev Physiol 1992;54:373-94.

5. Wallace MJ, Kitchen MJ, Lewis RA, et al. Inspiration regulates the rate and temporal pattern of lung liquid clearance and lung aeration at birth. J Appl Physiol (1985) 2009;106:1888-95.

6. Hooper SB, Te Pas AB, Kitchen MJ. Respiratory transition in the newborn: a three-phase process. Arch Dis Child Fetal Neonatal Ed. 2016;101(3): F266-71.

7. Hooper SB, Te Pas AB, Lang J, et al. Cardiovascular transition at birth: a physiological sequence. Pediatr Res 2015;77:608-14.

8. Roberts D, Dalziel S. Antenatal corticosteroids for accelerating fetal lung maturation for women at risk of preterm birth. Cochrane Database Syst Rev 2006;3:CD004454.

9. Stock KW, Chen Q, Hatabu H, Edelman RR. Magnetic resonance T2* measurements of the normal human lung in vivo with ultra-short echo times. Magn Reson Imaging 1999;17:997-1000.

10. Viard R, Vermandel M, Tourneux P, Storme L, Rousseau J. Lung liquid clearance in newborn lamb: MRI methods and preliminary results. Conf Proc IEEE Eng Med Biol Soc 2007;2007:2891-4.

11. Viard R, Tourneux P, Storme L, Girard JM, Betrouni N, Rousseau J. Magnetic resonance imaging spatial and time study of lung water content in newborn lamb: methods and preliminary results. Invest Radiol 2008;43:470-80.

12. Storme L, Riou Y, Leclerc F, et al. Respiratory mechanics in mechanically ventilated newborns: a comparison between passive inflation and occlusion methods. Pediatr Pulmonol 1992;12:203-12.

13. Riou Y, Storme L, Leclerc F, Neve V, Logier R, Lequien P. Comparison of four methods for measuring elevation of FRC in mechanically ventilated infants. Intensive Care Med 1999;25:1118-25.

14. Miserocchi G, Poskurica BH, Del Fabbro M. Pulmonary interstitial pressure in anesthetized paralyzed newborn rabbits. J Appl Physiol (1985) 1994;77:2260-8.

15. Sundell HW, Harris TR, Cannon JR, et al. Lung water and vascular permeability-surface area in premature newborn lambs with hyaline membrane disease. Circ Res 1987;60:923-32.

16. Alcorn DG, Adamson TM, Maloney JE, Robinson PM. A morphologic and morphometric analysis of fetal lung development in the sheep. Anat Rec 1981;201:655-67.

17. Bland RD, Carlton DP, Scheerer RG, Cummings JJ, Chapman DL. Lung fluid balance in lambs before and after premature birth. J Clin Invest 1989;84:568-76. 
18. Chapman DL, Carlton DP, Nielson DW, Cummings JJ, Poulain FR, Bland RD. Changes in lung lipid during spontaneous labor in fetal sheep. J Appl Physiol (1985) 1994;76:523-30.

19. Wilson SM, Olver RE, Walters DV. Developmental regulation of lumenal lung fluid and electrolyte transport. Respir Physiol Neurobiol 2007;159:247-55.

20. Bland RD. Pathogenesis of pulmonary edema after premature birth. Adv Pediatr 1987;34:175-221.

21. Bland RD, Boyd CA. Cation transport in lung epithelial cells derived from fetal, newborn, and adult rabbits. J Appl Physiol (1985) 1986;61:507-15.

22. Humphreys PW, Normand IC, Reynolds EO, Strang LB. Pulmonary lymph flow and the uptake of liquid from the lungs of the lamb at the start of breathing. J Physiol 1967;193:1-29.

23. Gyamfi-Bannerman C, Thom EA, Blackwell SC, et al.; NICHD MaternalFetal Medicine Units Network. Antenatal betamethasone for women at risk for late preterm delivery. N Engl J Med 2016;374:1311-20.

24. Sotiriadis A, Makrydimas G, Papatheodorou S, et al. Corticosteroids for preventing neonatal respiratory morbidity after elective caesarean section at term. Cochrane Database Syst Rev 2009;7;(4):CD006614.

25. Stutchfield P, Whitaker R, Russell I; Antenatal Steroids for Term Elective Caesarean Section (ASTECS) Research Team. Antenatal betamethasone and incidence of neonatal respiratory distress after elective caesarean section: pragmatic randomised trial. BMJ 2005;331:662.

26. Rebello CM, Ikegami M, Ervin MG, Polk DH, Jobe AH. Postnatal lung function and protein permeability after fetal or maternal corticosteroids in preterm lambs. J Appl Physiol (1985) 1997;83:213-8.

27. Riou Y, Leclerc F, Scalfaro P, Abazine A, Neve V, Storme L. Effect of increasing inspiratory time on respiratory mechanics in mechanically ventilated neonates. Pediatr Crit Care Med 2002;3:45-51.

28. Estilaei M, MacKay A, Roberts C, Mayo J. 1H NMR measurements of wet/ dry ratio and T1, T2 distributions in lung. J Magn Reson 1997;124:410-9.

29. Estilaei M, MacKay A, Whittall K, Mayo J. In vitro measurements of water content and T2 relaxation times in lung using a clinical MRI scanner. J Magn Reson Imaging 1999;9:699-703.
30. Holverda S, Theilmann RJ, Sá RC, et al. Measuring lung water: ex vivo validation of multi-image gradient echo MRI. J Magn Reson Imaging 2011;34:220-4.

31. Mayo JR, MacKay AL, Whittall KP, Baile EM, Paré PD. Measurement of lung water content and pleural pressure gradient with magnetic resonance imaging. J Thorac Imaging 1995;10:73-81.

32. Cutillo AG, Morris AH, Blatter DD, et al. Determination of lung water content and distribution by nuclear magnetic resonance. J Appl Physiol Respir Environ Exerc Physiol 1984;57:583-8.

33. Caruthers SD, Paschal CB, Pou NA, Roselli RJ, Harris TR. Regional measurements of pulmonary edema by using magnetic resonance imaging. J Appl Physiol (1985) 1998;84:2143-53.

34. Kuethe DO, Filipczak PT, Hix JM et al. Magnetic resonance imaging provide sensitive in vivo assessment of experimental ventilatorinduced lung injury. Am J Physiol Lung Cell Mol Physiol 2016;31: 208-18.

35. Walkup LL, Tkach JA, Higano NS, et al. Quantitative magnetic resonance imaging of bronchopulmonary dysplasia in the neonatal intensive care unit environment. Am J Respir Crit Care Med 2015;192:1215-22.

36. Adams EW, Counsell SJ, Hajnal JV, et al. Magnetic resonance imaging of lung water content and distribution in term and preterm infants. Am J Respir Crit Care Med 2002;166:397-402.

37. Theilmann RJ, Darquenne C, Elliott AR, Bailey BA, Conrad DJ. Characterizing lung disease in cystic fibrosis with with magnetic resonance imaging and airway physiology. PLoS One. 2016;23:1133.

38. Lange NR, Schuster DP. The measurement of lung water. Crit Care 1999;3:R19-24.

39. Bland RD, Bressack MA, McMillan DD. Labor decreases the lung water content of newborn rabbits. Am J Obstet Gynecol 1979;135: 364-7.

40. Bland RD, McMillan DD, Bressack MA, Dong L. Clearance of liquid from lungs of newborn rabbits. J Appl Physiol Respir Environ Exerc Physiol 1980;49:171-7. 